\title{
14. AMINO ACIDS IN THE INTERSTITIAL WATERS FROM ODP SITE 695 IN THE WEDDELL SEA, ANTARCTIC OCEAN ${ }^{1}$
}

\author{
Hodaka Kawahata, ${ }^{2}$ Toshio Ishizuka, ${ }^{3}$ and Toshiyasu Nagao ${ }^{4}$
}

\begin{abstract}
Site 695 lies on the southeast margin of the South Orkney microcontinent on the northern margin of the Weddell Sea, at $62^{\circ} 23.48^{\prime} \mathrm{S}, 43^{\circ} 27.10^{\prime} \mathrm{W}$ in $1305 \mathrm{~m}$ water depth. The inorganic properties of interstitial waters at this site, including sulfate reduction, biogenic methane production, and high concentrations of ammonia and phosphate, imply high microbial activity. However, no clear relationship between amino acid composition and concentration and the type of microbial activity (e.g., sulfate reduction or methane production) can be identified.

The THAA (total hydrolyzable amino acids) values range between 2.45 and $17.31 \mu \mathrm{mol} / \mathrm{L}$, averaging $7.14 \mu \mathrm{mol} / \mathrm{L}$. The mean concentrations and relative abundance values of acidic, basic, neutral, aromatic, and sulfur-containing amino acids are $1.34(18 \%), 1.09(15 \%), 3.93(54 \%), 0.50(8 \%)$, and $0.02(0 \%) \mu \mathrm{mol} / \mathrm{L}$, respectively. Glycine is the most abundant amino acid residue, with serine, glutamic acid, and ornithine next. The DFAA (dissolved free amino acids) values range from 0.10 to $12.73 \mu \mathrm{mol} / \mathrm{L}$, averaging $4.07 \mu \mathrm{mol} / \mathrm{L}$. The acidic, basic, neutral, aromatic, and sulfurcontaining amino acids are on average $0.21,0.79,2.56,0.41$, and $0.01 \mu \mathrm{mol} / \mathrm{L}$, respectively. The relative abundances of acidic, basic, neutral, and aromatic amino acids average $4 \%, 18 \%, 58 \%$, and $15 \%$, respectively.

Predominance of DFAA over DCAA (dissolved combined amino acids) in interstitial waters of Lithologic Units I and II is contrary to the predominance of DCAA over DFAA in other interstitial waters and seawater.

The comparison of amino acid compositions between DCAA and siliceous plankton suggests that the DCAA in interstitial waters originally comes from amino acids derived from siliceous plankton. However, other sources which are much enriched in glutamic acid contribute to the DCAA composition.
\end{abstract}

\section{INTRODUCTION}

Amino acids are one of the most important organic compounds in marine sediments and interstitial waters. They undergo decomposition and transformation during early diagenesis. Although interstitial waters originate mainly from seawater, the concentration of amino acids in interstitial waters are one to three orders of magnitude higher than that of overlying seawater and the composition of interstitial water is different from that of seawater (e.g., Lee and Bada, 1975; Michaelis et al., 1982; Henrichs and Farrington, 1984, 1987; Ishizuka et al., 1988). Dissolved amino acids in interstitial waters are very sensitive indicators of the chemical and biological processes occurring in the sedimentary column. However, detailed analyses of dissolved free and total hydrolyzable amino acids (DFAA and THAA) in interstitial waters have not, until this study, been extended to sediments buried deeper than $30 \mathrm{~m}$ (Kawahata and Ishizuka, 1989).

Gradients in inorganic ion concentrations have been used as indicators of diagenesis of organic matter in deep sediments and interstitial waters. Sulfate depletion and maxima in ammonia concentrations were interpreted to be a consequence of sulfate reduction reactions (Manheim and Schug, 1978). Hole 695A provides a good opportunity to study early diagenesis of organic materials. The reasons are that a high sedimentation rate, due to the high diatom productivity, is observed in the upper $200 \mathrm{~m}$ thick section; that microbiological activity, especially sulfate reduction and methanogenesis, is evidenced by the intersti-

\footnotetext{
${ }^{1}$ Barker, P. F., Kennett, J. P., et al., 1990. Proc. ODP, Sci. Results, 113: College Station, TX (Ocean Drilling Program).

2 Geological Survey of Japan, Higashi, Tsukuba, Ibaraki, 305, Japan. Japan.

3 Ocean Research Institute, University of Tokyo, Minamidai, Nakano, Tokyo,

${ }^{4}$ Kanazawa University, Marunouchi, Kanazawa 920, Japan.
}

tial water chemistry; and that fairly high concentrations of ammonia and phosphate, related to biological activity, are found.

The purpose of this study was to determine the vertical distribution of DFAA and THAA in interstitial waters and to discuss the chemical and biological processes affecting the compositions of dissolved free and combined amino acids in interstitial waters.

\section{METHODS}

\section{Sampling and storage}

Interstitial waters of Site 695 sediments were collected on the JOIDES Resolution by hydraulic squeezing. All interstitial water samples were filtered through $0.22-\mu \mathrm{m}$ Millipore cellulose acetate filters in the shipboard laboratory. For amino acid analysis in interstitial water, a $5 \mathrm{~mL}$ aliquot of the water sample was placed into a precombusted glass ampule. The ampule was sealed after flushing with helium, stored in a refrigerator $\left(1^{\circ}-2^{\circ} \mathrm{C}\right)$, and returned to the land-based laboratory.

\section{Analytical procedures}

\section{Amino acids}

Dissolved free amino acids (DFAA) in interstitial water were analyzed after the addition of $7 \mu \mathrm{L}$ of ultrapure (amino acid free) $17 \% \mathrm{HCl}$ with $0.7 \mathrm{~mL}$ of an interstitial water sample. Amino acid concentrations were determined by direct injection of the sample into an automated liquid chromatograph (Hitachi Model 835). Reported concentrations have been corrected for the reagent blank.

For total hydrolyzable amino acids (THAA), $1 \mathrm{~mL}$ of the interstitial water, together with ultrapure concentrated $\mathrm{HCI}$, was adjusted to $6 \mathrm{~N}$ and placed into a precombusted glass ampule and hydrolyzed at $110^{\circ} \mathrm{C}$ for $22 \mathrm{hr}$ under an argon atmosphere. Then, the solution was gently evaporated using a rotary evaporator at a temperature lower than $42^{\circ} \mathrm{C}$ and the residue redis- 
solved in $1 \mathrm{~mL}$ of $0.01 \mathrm{~mol} \mathrm{HCl}$. This aliquot was then injected into the amino acid analyzer. The standard deviation for amino acid analysis based on replicated measurements of a standard solution (about $1 \mu \mathrm{mol} / \mathrm{L}$ for amino acids) was less than $10 \%$ and the detection limit of our method was about $0.05 \mu \mathrm{mol} / \mathrm{L}$. Low values in DFAA and THAA near the detection limit may not be dependable. Nevertheless, we retain these values because they confirm that the amino acid concentration is low (Ishizuka et al., 1988).

\section{SUMMARY OF GEOLOGICAL FRAMEWORK}

Site 695 lies on the southeast margin of the South Orkney microcontinent on the northern margin of the Weddell Sea, at $62^{\circ} 23.48^{\prime} \mathrm{S}, 43^{\circ} 27.10^{\prime} \mathrm{W}$ in $1305 \mathrm{~m}$ water depth (Fig. 1). Three lithologic units were identified: Unit I consists of diatom-rich (up to $90 \%$ ) oozes and silty, clayey muds of Quaternary age; Unit II consists of diatom-bearing silty and clayey mud $(10 \%-$ $25 \%$ biosiliceous) of lower Pliocene age; Unit III consists of silty muds of lower Pliocene and likely uppermost Miocene (Table 1). Ice-rafted detritus most commonly occurs above $20 \mathrm{~m}$ sub-bottom depth and Unit II (Shipboard Scientific Party, 1988).

The relative abundance of clay minerals is on average $31 \%$ throughout the hole. Illite and chlorite are the dominant clay minerals at Site 695 . Kaolinite is rare to common in Unit I but uniformly rare beneath. Smectite abundance is variable. Total

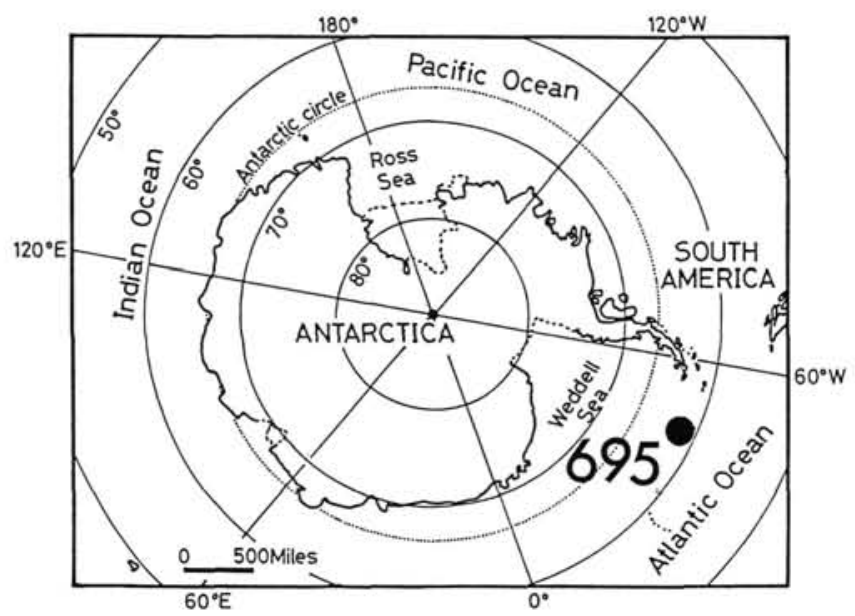

Figure 1. Location of ODP Site 695 in the Weddell Sea. organic carbon (TOC) values range from 0.10 to $0.67 \mathrm{wt} \%$, averaging 0.38 wt \% (Shipboard Scientific Party, 1988).

The sedimentation rate is on average less than $5 \mathrm{~m} / \mathrm{m}$.y. for the Pleistocene, based upon paleomagnetic data. Paleomagnetic and biostratigraphic data indicate that sedimentation rates for the Pliocene and the uppermost Miocene are $90 \mathrm{~m} / \mathrm{m}$.y. from 18 to $90 \mathrm{~m}$ sub-bottom depth, $215 \mathrm{~m} / \mathrm{m}$.y. down to $245 \mathrm{~m}$, and 105 m/m.y. down to $330 \mathrm{~m}$ (Shipboard Scientific Party, 1988; table 1).

\section{INORGANIC CHEMISTRY OF INTERSTITIAL WATERS}

The chlorinity and $\mathrm{pH}$ values are around $563 \mathrm{mmol} / \mathrm{L}$ and 8.0 , respectively, throughout the hole. The concentration of phosphate is high, $4.8 \mu \mathrm{mol} / \mathrm{L}$ on average, as a result of high bacterial activity and high rates of sedimentation. The concentration of ammonia increases from $0.04 \mathrm{mmol} / \mathrm{L}$ in the shallower section and peaks at $1.76 \mathrm{mmol} / \mathrm{L}$ at $260 \mathrm{~m}$ sub-bottom depth, below which only minor variations take place. The almost stable level below $260 \mathrm{~m}$ sub-bottom depth represents the depth where the rate of processes removing ammonia from the interstitial water equals or exceeds the rate at which ammonia is generated microbially (Shipboard Scientific Party, 1988).

The concentration of sulfate is constant at about seawater values $(29 \mathrm{mmol} / \mathrm{L})$ in the upper $50 \mathrm{~m}$ sub-bottom depth and decreases linearly to zero at $340 \mathrm{~m}$, which is related to high primary production of organic matter, its escaping oxidation in the water column, and high sedimentation rates. The presence of considerable amounts of sulfides resulted from sulfate reduction. The nitrate concentration is estimated to be quite low in this core because the sulfate reduction stage, in general, follows the nitrate reduction stage, and because intense sulfate reduction is found in this core. Methane concentrations increase sharply below $250 \mathrm{~m}$ sub-bottom depth, near the base of the pore-water sulfate ion depletion curve, suggesting that the methane is biogenic and that ample organic matter sustains microbial activity below $250 \mathrm{~m}$ sub-bottom depth (Shipboard Scientific Party, 1988).

\section{RESULTS}

Total hydrolyzable amino acid (THAA) concentrations are given in Table 2 and plotted versus sub-bottom depth in Figure 2. The THAA values range between 2.45 and $17.31 \mu \mathrm{mol} / \mathrm{L}$, averaging $7.14 \mu \mathrm{mol} / \mathrm{L}$. Relatively high values are found at the nearsurface, about $50 \mathrm{~m}, 180 \mathrm{~m}$, and $320 \mathrm{~m}$ sub-bottom depth. A discrepancy between the organic carbon content of the sediment and the THAA and DFAA profiles of interstitial waters is found in Hole 695A (Fig. 2). The exchange between organic matter in the sediment and THAA and DFAA in interstitial waters seems

Table 1. Characteristics of each sedimentary zone of Hole 695A.

\begin{tabular}{|c|c|c|c|c|c|}
\hline & nit & Depth & Lithology & Age & $\begin{array}{c}\text { Average } \\
\text { porosity and } \\
\text { bulk density }\end{array}$ \\
\hline \multirow[t]{4}{*}{ I } & IA & $0-19.9 \mathrm{~m}$ & $\begin{array}{l}\text { Diatom-bearing silty and clayey muds } \\
\text { with minor foraminifers }\end{array}$ & $\begin{array}{l}\text { Quaternary- } \\
\text { middle Pliocene }\end{array}$ & $\begin{array}{l}61 \% \\
1.4 \mathrm{~g} / \mathrm{cm}^{3}\end{array}$ \\
\hline & IB & $19.9-52.3 \mathrm{~m}$ & Muddy diatom oozes & $\begin{array}{l}\text { middle Pliocene- } \\
\text { early Pliocene }\end{array}$ & $\begin{array}{l}77 \% \\
1.5 \mathrm{~g} / \mathrm{cm}^{3}\end{array}$ \\
\hline & IC & $52.3-93.7 \mathrm{~m}$ & Silty and muddy diatom oozes & early Pliocene & $\begin{array}{l}68 \% \\
1.6 \mathrm{~g} / \mathrm{cm}^{3}\end{array}$ \\
\hline & ID & $93.7-190.7 \mathrm{~m}$ & Diatom oozes to diatom silty muds & early Pliocene & $\begin{array}{l}75 \% \\
1.5 \mathrm{~g} / \mathrm{cm}^{3}\end{array}$ \\
\hline & II & $190.7-306.9 \mathrm{~m}$ & Diatom-bearing silty and clayey muds & early Pliocene & $\begin{array}{l}58 \% \\
1.9 \mathrm{~g} / \mathrm{cm}^{3}\end{array}$ \\
\hline & III & $306.9-341.1 \mathrm{~m}$ & Silty mud & $\begin{array}{l}\text { early Pliocene- } \\
\text { latest Miocene }\end{array}$ & $\begin{array}{l}44 \% \\
2.1 \mathrm{~g} / \mathrm{cm}^{3}\end{array}$ \\
\hline
\end{tabular}


Table 2. Concentrations in $\mu \mathrm{mol} / \mathrm{L}$ of DFAA in interstitial waters from Hole 695A.

\begin{tabular}{|c|c|c|c|c|c|c|c|c|c|c|c|c|c|c|}
\hline $\begin{array}{l}\text { Core-Section } \\
\text { Interval (cm) } \\
\text { Depth (m) }\end{array}$ & $\begin{array}{c}1 \mathrm{H}-3 \\
145-150 \\
4.5\end{array}$ & $\begin{array}{c}3 \mathrm{H}-4 \\
120-125 \\
18.1\end{array}$ & $\begin{array}{c}6 \mathrm{H}-3 \\
120-125 \\
45.6\end{array}$ & $\begin{array}{c}9 \mathrm{H}-2 \\
120-125 \\
72.7\end{array}$ & $\begin{array}{c}12 \mathrm{H}-4 \\
120-125 \\
94.9\end{array}$ & $\begin{array}{c}15 \mathrm{H}-3 \\
120-125 \\
122.4\end{array}$ & $\begin{array}{c}19 X-4 \\
120-125 \\
153\end{array}$ & $\begin{array}{r}22 X-3 \\
120-125 \\
180.7\end{array}$ & $\begin{array}{c}25 X-3 \\
120-121 \\
200.1\end{array}$ & $\begin{array}{c}29 \mathrm{X}-2 \\
115-125 \\
237.4\end{array}$ & $\begin{array}{c}32 X-4 \\
115-125 \\
259.8\end{array}$ & $\begin{array}{c}36 X-2 \\
115-125 \\
295.5\end{array}$ & $\begin{array}{c}39 \mathrm{X}-3 \\
115-125 \\
316.2\end{array}$ & $\begin{array}{c}41 X-3 \\
140-150 \\
335.9\end{array}$ \\
\hline \multicolumn{15}{|l|}{ Acidic } \\
\hline $\begin{array}{l}\text { Aspartic acid } \\
\text { Glutamic acid }\end{array}$ & $\begin{array}{l}0.10 \\
0.08\end{array}$ & $\begin{array}{c}0.03 \\
-\end{array}$ & $\begin{array}{l}0.25 \\
0.08\end{array}$ & $\begin{array}{l}0.11 \\
0.04\end{array}$ & $\begin{array}{l}0.11 \\
-\end{array}$ & $\overline{-}$ & $\begin{array}{l}0.33 \\
0.04\end{array}$ & $\begin{array}{l}0.60 \\
0.18\end{array}$ & $\begin{array}{l}0.38 \\
0.09\end{array}$ & $\bar{z}$ & 0.08 & $\begin{array}{l}0.17 \\
-\end{array}$ & $\begin{array}{l}0.12 \\
-\end{array}$ & $\begin{array}{l}0.07 \\
0.06\end{array}$ \\
\hline \multicolumn{15}{|l|}{ Basic } \\
\hline Ornithine & 0.44 & 0.21 & 0.77 & 0.35 & 0.23 & - & 0.66 & 1.56 & 1.36 & - & 0.16 & 0.99 & 0.44 & - \\
\hline Lysine & 0.13 & 0.06 & 0.19 & 0.15 & 0.12 & - & 0.20 & 0.39 & 0.28 & - & 0.06 & 0.17 & 0.06 & 0.05 \\
\hline Histidine & - & - & - & $\overline{0}$ & $\overline{-17}$ & - & - & - & $\overline{-1}$ & $\overline{0.15}$ & - & $\overline{10}$ & $\overline{0}$ & $\overline{-}$ \\
\hline Arginine & 0.15 & 0.10 & - & 0.35 & 0.37 & - & 0.13 & 0.15 & 0.24 & 0.15 & - & 0.18 & 0.19 & \\
\hline \multicolumn{15}{|l|}{ Neutral } \\
\hline \multicolumn{15}{|l|}{ hydroxy } \\
\hline Threonine & 0.08 & - & 0.27 & 0.15 & 0.15 & - & 0.35 & 0.67 & 0.42 & 0.00 & 0.10 & 0.19 & 0.13 & 0.06 \\
\hline Serine & 1.03 & 0.22 & 1.26 & 0.47 & 0.51 & - & 1.73 & 3.24 & 2.09 & 0.06 & 0.46 & 0.87 & 0.67 & 0.34 \\
\hline \multicolumn{15}{|l|}{ straight } \\
\hline Glycine & 0.72 & 0.31 & 0.98 & 0.55 & 0.47 & 0.05 & 1.35 & 2.19 & 1.49 & 0.10 & 0.41 & 0.68 & 0.67 & 0.33 \\
\hline Alanine & 0.43 & 0.04 & - & 0.30 & 0.24 & - & 0.72 & 1.28 & 0.80 & 0.04 & 0.22 & 0.41 & 0.40 & 0.14 \\
\hline \multicolumn{15}{|l|}{ branch } \\
\hline Valine & - & - & 0.12 & - & - & - & - & 0.42 & 0.15 & 0.03 & - & - & - & - \\
\hline Iso-leucine & 0.10 & - & 0.17 & 0.05 & 0.04 & - & 0.15 & 0.26 & 0.17 & - & - & 0.12 & 0.12 & 0.04 \\
\hline Leucine & 0.08 & - & 0.24 & 0.29 & 0.04 & - & 0.11 & 0.31 & 0.24 & - & - & - & - & - \\
\hline \multicolumn{15}{|l|}{ The rest } \\
\hline Aspargine & - & - & 0.13 & 0.12 & - & - & 0.21 & 0.54 & 0.21 & 0.21 & - & - & 0.31 & - \\
\hline \multicolumn{15}{|l|}{ Aromatic } \\
\hline Tyrosine & - & 0.38 & 0.43 & - & 0.36 & - & 0.32 & 0.43 & 0.43 & - & 0.40 & 0.47 & 0.44 & 0.42 \\
\hline Phenylalanine & - & 0.04 & 0.31 & 0.05 & 0.04 & 0.05 & 0.22 & 0.23 & 0.30 & 0.01 & - & 0.08 & 0.02 & 0.26 \\
\hline Sulfur & & & & & - & & & & & & & & & \\
\hline Methionine & - & - & - & - & - & - & 0.07 & 0.04 & 0.07 & - & - & - & - & - \\
\hline \multicolumn{15}{|l|}{ The rest } \\
\hline Taurine & - & - & - & 0.01 & - & - & 0.02 & 0.20 & 0.02 & 0.02 & - & 0.01 & 0.02 & 0.01 \\
\hline Citrulline & - & - & 0.04 & - & - & - & - & - & - & - & - & - & - & - \\
\hline Crytathionine & - & - & - & - & - & - & - & - & 0.06 & 0.01 & - & 0.09 & - & - \\
\hline$\alpha$-aminoadipic acid & - & - & - & 0.11 & 0.15 & - & - & - & - & 0.18 & - & - & - & - \\
\hline$\beta$-aminobutyric acid & $\overline{0.23}$ & $\overline{-}$ & $\overline{-}$ & $\overline{-}$ & $\overline{-}$ & - & $\overline{-}$ & $\overline{004}$ & - & - & - & - & - & $\overline{0}$ \\
\hline $\begin{array}{l}\beta \text {-aminobutyric acid } \\
\beta \text {-alanine }\end{array}$ & - & $\overline{-}$ & $\overline{-}$ & 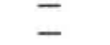 & $\overline{-}$ & $\overline{-}$ & $\bar{z}$ & $\begin{array}{l}0.04 \\
-\end{array}$ & $\overline{-}$ & $\bar{z}$ & $\bar{z}$ & $\bar{z}$ & $\overline{-}$ & $\begin{array}{l}0.15 \\
-\end{array}$ \\
\hline Total & $\overline{3.57}$ & $\overline{1.39}$ & 5.24 & $\overline{3.10}$ & $\overline{2.83}$ & $\overline{0.10}$ & $\overline{6.61}$ & $\overline{12.73}$ & 8.80 & $\overline{0.81}$ & $\overline{1.89}$ & $\overline{4.43}$ & $\overline{3.59}$ & $\overline{1.93}$ \\
\hline
\end{tabular}




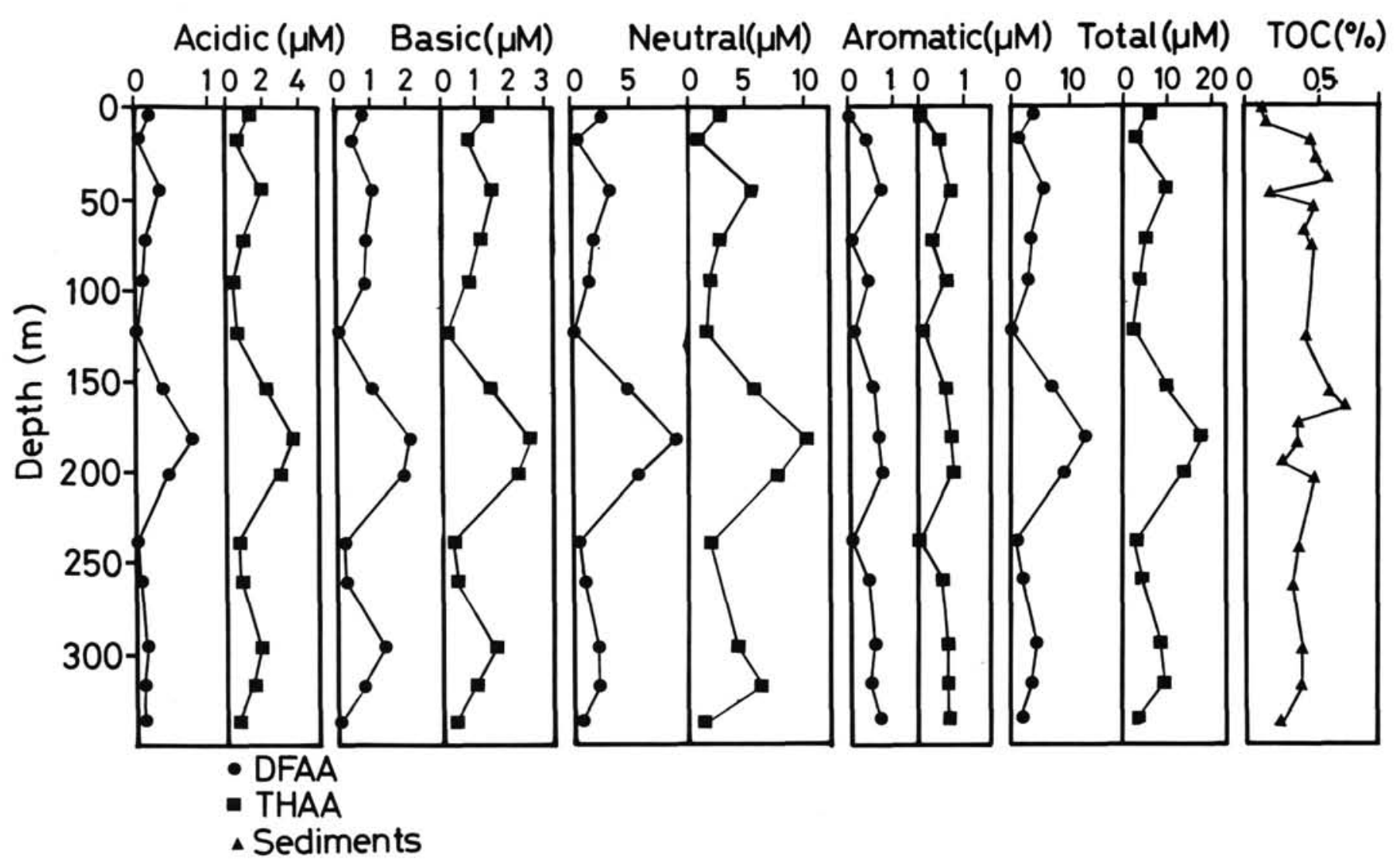

Figure 2. Concentration of acidic, basic, neutral, and total amino acids of THAA and DFAA in interstitial waters vs. sub-bottom depth in Hole 695A.

to be limited because even at greater depths the two profiles do not approach each other.

Neutral amino acids are the most abundant fraction of amino acids and account for $54 \%$ of the total THAA. The average concentration is $3.93 \mu \mathrm{mol} / \mathrm{L}$. The second most abundant fraction is acidic amino acids. The mean concentration is $1.34 \mu \mathrm{mol} / \mathrm{L}$, which constitutes $18 \%$ of the DFAA. The basic amino acid fraction is $1.09 \mu \mathrm{mol} / \mathrm{L}$ on average, which accounts for $15 \%$ of THAA. The aromatic and sulfur-containing amino acids are minor fractions. Their mean values are 0.50 and $0.02 \mu \mathrm{mol} / \mathrm{L}$, respectively. The relative abundance of aromatic amino acids is only $8 \%$ of THAA. THAA composition shows no consistent trend with sub-bottom depth, nor with the total concentration.

Glycine is the most abundant amino acid residue, with serine, glutamic acid, and ornithine next. Trace concentrations of the nonprotein amino acids, $\beta$-amino butyric acid and $\beta$-alanine constitute about 1.6 and $1.5 \%$ of THAA, respectively. Low levels of $\alpha$-amino adipic acid are also present. However, $\alpha$-amino butyric acid, which is the usual amino acid in sediments, is not found in THAA in interstitial waters.

Dissolved free amino acid (DFAA) concentrations in interstitial waters are given in Table 3. The DFAA values range from 0.10 to $12.73 \mu \mathrm{mol} / \mathrm{L}$, averaging $4.07 \mu \mathrm{mol} / \mathrm{L}$. The acidic, basic, neutral, aromatic, and sulfur-containing amino acids are on average $0.21,0.79,2.56,0.41$, and $0.01 \mu \mathrm{mol} / \mathrm{L}$, respectively. The relative abundances of acidic, basic, neutral, and aromatic amino acids average $4 \%, 18 \%, 58 \%$, and $15 \%$, respectively.
Figure 2 shows the DFAA concentration versus sub-bottom depth. The profile is similar to that of THAA.

\section{DISCUSSION}

\section{The Ratio of Combined Amino Acids to DFAA in Interstitial Waters}

The concentration of dissolved combined amino acids (DCAA) can be calculated by subtracting the DFAA concentration from the THAA concentration in interstitial waters from Hole 695A (Table 4). The concentrations of the DCAA and the DFAA are on average 2.7 and $4.5 \mu \mathrm{mol} / \mathrm{L}$ in Lithologic Unit I, 3.5, 4 $\mu \mathrm{mol} / \mathrm{L}$ in Unit II, and 3.7 and $2.8 \mu \mathrm{mol} / \mathrm{L}$ in Unit III, respectively. The results show predominance of DFAA over DCAA present in interstitial water in Units I and II, which is in contrast to previous results from interstitial waters and seawater.

The profile of total DCAA looks similar to those of the total THAA and DFAA. The reason is that the profiles of total DCAA, THAA, and DFAA are much affected by those of their neutral amino acid fractions, since they account for more than $50 \%$ of relative abundance of amino acids. Kawahata and Ishizuka (1989) found greater concentrations of DCAA than DFAA in interstitial water from biogenic carbonate and silica-rich sediments from the Panama Basin. The mean concentrations of DCAA and DFAA are 5.1 and $3.2 \mu \mathrm{mol} / \mathrm{L}$, respectively. Lee and Bada (1975) found that there are about ten times more combined than free amino acids in seawater. The DFAA from open 
Table 3. Concentrations in $\mu \mathrm{mol} / \mathrm{L}$ of THAA in interstitial waters from Hole 695A.

\begin{tabular}{|c|c|c|c|c|c|c|c|c|c|c|c|c|c|c|}
\hline $\begin{array}{c}\text { Core-Section } \\
\text { Interval }(\mathrm{cm}) \\
\text { Depth }(\mathrm{m})\end{array}$ & $\begin{array}{c}1 \mathrm{H}-3 \\
145-150 \\
4.5\end{array}$ & $\begin{array}{c}3 \mathrm{H}-4 \\
120-125 \\
18.1\end{array}$ & $\begin{array}{c}6 \mathrm{H}-3 \\
120-125 \\
45.6\end{array}$ & $\begin{array}{c}9 \mathrm{H}-2 \\
120-125 \\
72.7\end{array}$ & $\begin{array}{c}12 \mathrm{H}-4 \\
120-125 \\
94.9\end{array}$ & $\begin{array}{c}15 \mathrm{H}-3 \\
120-125 \\
122.4\end{array}$ & $\begin{array}{c}19 \mathrm{X}-4 \\
120-125 \\
153\end{array}$ & $\begin{array}{r}22 X-3 \\
120-125 \\
180.7\end{array}$ & $\begin{array}{r}25 X-3 \\
120-121 \\
200.1\end{array}$ & $\begin{array}{c}29 \mathrm{X}-2 \\
115-125 \\
237.4\end{array}$ & $\begin{array}{c}32 X-4 \\
115-125 \\
259.8\end{array}$ & $\begin{array}{c}36 \mathrm{X}-2 \\
115-125 \\
295.5\end{array}$ & $\begin{array}{c}39 X-3 \\
115-125 \\
316.2\end{array}$ & $\begin{array}{c}41 X-3 \\
140-150 \\
335.9\end{array}$ \\
\hline \multicolumn{15}{|l|}{ Acidic } \\
\hline $\begin{array}{l}\text { Aspartic acid } \\
\text { Glutamic acid }\end{array}$ & $\begin{array}{l}0.13 \\
1.03\end{array}$ & $\begin{array}{l}0.14 \\
0.38\end{array}$ & $\begin{array}{l}0.53 \\
1.32\end{array}$ & $\begin{array}{l}0.23 \\
0.56\end{array}$ & $\begin{array}{l}0.13 \\
0.27\end{array}$ & $\begin{array}{l}0.18 \\
0.28\end{array}$ & $\begin{array}{l}0.53 \\
1.52\end{array}$ & $\begin{array}{l}0.99 \\
2.66\end{array}$ & $\begin{array}{l}0.87 \\
2.01\end{array}$ & $\begin{array}{l}0.16 \\
0.44\end{array}$ & $\begin{array}{l}0.23 \\
0.51\end{array}$ & $\begin{array}{l}0.44 \\
1.33\end{array}$ & $\begin{array}{l}0.48 \\
0.94\end{array}$ & $\begin{array}{l}0.17 \\
0.34\end{array}$ \\
\hline \multicolumn{15}{|l|}{ Basic } \\
\hline $\begin{array}{l}\text { Ornithine } \\
\text { Lysine } \\
\text { Histidine } \\
\text { Arginine }\end{array}$ & $\begin{array}{l}0.80 \\
0.39 \\
\overline{0.15}\end{array}$ & $\begin{array}{l}0.51 \\
0.18 \\
-\overline{0.10}\end{array}$ & $\begin{array}{l}0.99 \\
0.27 \\
\overline{0.15}\end{array}$ & $\begin{array}{l}0.50 \\
0.28 \\
\overline{0.35}\end{array}$ & $\begin{array}{l}0.28 \\
0.13 \\
-\overline{0}\end{array}$ & $\begin{array}{l}0.09 \\
0.09 \\
- \\
-\end{array}$ & $\begin{array}{l}0.93 \\
0.29 \\
\overline{0.13}\end{array}$ & $\begin{array}{l}1.83 \\
0.55 \\
- \\
0.15\end{array}$ & $\begin{array}{l}1.61 \\
0.32 \\
\overline{0.24}\end{array}$ & $\begin{array}{l}0.11 \\
0.08 \\
\overline{0.15}\end{array}$ & $\begin{array}{l}0.18 \\
0.10 \\
- \\
0.13\end{array}$ & $\begin{array}{l}0.99 \\
0.34 \\
\overline{0.18}\end{array}$ & $\begin{array}{l}0.60 \\
0.15 \\
\overline{0.19}\end{array}$ & $\begin{array}{c}0.29 \\
0.08 \\
- \\
-\end{array}$ \\
\hline \multicolumn{14}{|l|}{ Neutral } & hydroxy \\
\hline $\begin{array}{l}\text { Threonine } \\
\text { Serine }\end{array}$ & $\begin{array}{l}0.08 \\
1.09\end{array}$ & $\overline{0.38}$ & $\begin{array}{l}0.32 \\
1.85\end{array}$ & $\begin{array}{l}0.15 \\
0.70\end{array}$ & $\begin{array}{l}0.15 \\
0.53\end{array}$ & $\begin{array}{l}0.08 \\
0.39\end{array}$ & $\begin{array}{l}0.40 \\
1.92\end{array}$ & $\begin{array}{l}0.74 \\
3.51\end{array}$ & $\begin{array}{l}0.60 \\
2.90\end{array}$ & $\begin{array}{l}0.11 \\
0.30\end{array}$ & $\begin{array}{l}0.17 \\
0.69\end{array}$ & $\begin{array}{l}0.26 \\
1.21\end{array}$ & $\begin{array}{l}0.44 \\
2.13\end{array}$ & $\begin{array}{l}0.11 \\
0.34\end{array}$ \\
\hline \multicolumn{15}{|l|}{ straight } \\
\hline $\begin{array}{l}\text { Glycine } \\
\text { Alanine }\end{array}$ & $\begin{array}{l}0.96 \\
0.43\end{array}$ & $\begin{array}{l}0.52 \\
0.04\end{array}$ & $\begin{array}{l}1.80 \\
0.63\end{array}$ & $\begin{array}{l}1.12 \\
0.34\end{array}$ & $\begin{array}{l}0.73 \\
0.24\end{array}$ & $\begin{array}{l}0.77 \\
0.24\end{array}$ & $\begin{array}{l}1.83 \\
0.76\end{array}$ & $\begin{array}{l}2.82 \\
1.28\end{array}$ & $\begin{array}{l}2.22 \\
0.92\end{array}$ & $\begin{array}{l}0.76 \\
0.37\end{array}$ & $\begin{array}{l}0.97 \\
0.39\end{array}$ & $\begin{array}{l}1.34 \\
0.92\end{array}$ & $\begin{array}{l}1.85 \\
0.89\end{array}$ & $\begin{array}{l}0.66 \\
0.23\end{array}$ \\
\hline \multicolumn{15}{|l|}{ branch } \\
\hline $\begin{array}{l}\text { Valine } \\
\text { Iso-leucine } \\
\text { Leucine }\end{array}$ & $\begin{array}{l}\overline{0.10} \\
0.16\end{array}$ & $\begin{array}{l}\overline{0.07} \\
-\end{array}$ & $\begin{array}{l}0.14 \\
0.20 \\
0.35\end{array}$ & $\begin{array}{l}\overline{0.10} \\
0.29\end{array}$ & $\begin{array}{l}\overline{0.07} \\
0.11\end{array}$ & $\begin{array}{l}-\overline{07} \\
0.07\end{array}$ & $\begin{array}{l}0.13 \\
0.18 \\
0.18\end{array}$ & $\begin{array}{l}0.51 \\
0.30 \\
0.35\end{array}$ & $\begin{array}{l}0.34 \\
0.22 \\
0.28\end{array}$ & $\begin{array}{l}0.03 \\
0.07 \\
0.07\end{array}$ & $\begin{array}{l}\overline{0} \\
0.11\end{array}$ & $\begin{array}{l}0.09 \\
0.13 \\
0.17\end{array}$ & $\begin{array}{l}0.23 \\
0.19 \\
0.18\end{array}$ & $\begin{array}{l}\overline{0.04} \\
0.06\end{array}$ \\
\hline \multicolumn{15}{|l|}{ The rest } \\
\hline Aspargine & - & - & 0.13 & 0.12 & - & - & 0.21 & 0.54 & 0.21 & 0.21 & 0.03 & 0.05 & 0.31 & - \\
\hline \multicolumn{15}{|l|}{ Aromatic } \\
\hline $\begin{array}{l}\text { Tyrosine } \\
\text { Phenylalanine }\end{array}$ & $\overline{-}$ & $\begin{array}{l}0.43 \\
0.04\end{array}$ & $\begin{array}{l}0.43 \\
0.32\end{array}$ & $\overline{0.29}$ & $\begin{array}{l}0.36 \\
0.29\end{array}$ & $\overline{0.05}$ & $\begin{array}{l}0.32 \\
0.29\end{array}$ & $\begin{array}{l}0.43 \\
0.31\end{array}$ & $\begin{array}{l}0.43 \\
0.37\end{array}$ & $\overline{0.01}$ & $\begin{array}{l}0.40 \\
0.16\end{array}$ & $\begin{array}{l}0.47 \\
0.22\end{array}$ & $\begin{array}{l}0.44 \\
0.22\end{array}$ & $\begin{array}{l}0.42 \\
0.26\end{array}$ \\
\hline \multicolumn{15}{|l|}{ Sulfur } \\
\hline \multicolumn{15}{|l|}{ The rest } \\
\hline $\begin{array}{l}\text { Taurine } \\
\text { Citrulline } \\
\text { Crytathionine } \\
\alpha \text {-aminoadipic acid } \\
\alpha \text {-aminobutyric acid } \\
\beta \text {-aminobutyric acid } \\
\beta \text {-alanine }\end{array}$ & $\begin{array}{l}\bar{z} \\
\bar{z} \\
\overline{-} \\
0.64 \\
=\end{array}$ & $\begin{array}{l}\bar{z} \\
\overline{-} \\
\overline{\overline{0}} \\
\overline{0}\end{array}$ & $\begin{array}{l}\overline{0.04} \\
= \\
\overline{-} \\
\overline{0}\end{array}$ & $\begin{array}{l}0.01 \\
\overline{-} \\
0.11 \\
\overline{0.06} \\
0.09\end{array}$ & $\begin{array}{l}\bar{z} \\
\overline{0} \\
\overline{0 .} \\
\overline{0.11}\end{array}$ & $\begin{array}{l}\overline{-} \\
= \\
\bar{z} \\
\overline{0.14}\end{array}$ & $\begin{array}{l}0.02 \\
= \\
= \\
\overline{-} \\
0.05 \\
0.08\end{array}$ & $\begin{array}{l}0.20 \\
= \\
= \\
\overline{0.04} \\
0.06\end{array}$ & $\begin{array}{l}0.02 \\
\overline{0.10} \\
= \\
\overline{-} \\
\overline{0.07}\end{array}$ & $\begin{array}{l}0.02 \\
-\overline{0} \\
0.01 \\
0.18 \\
- \\
0.07 \\
0.15\end{array}$ & $\begin{array}{l}0.02 \\
- \\
= \\
\overline{-} \\
0.03 \\
0.06\end{array}$ & $\begin{array}{l}0.02 \\
\overline{0} \\
\overline{0.14} \\
\overline{-} \\
0.09 \\
0.04\end{array}$ & $\begin{array}{l}0.05 \\
\overline{0.15} \\
\overline{-} \\
\overline{0.07} \\
0.04\end{array}$ & $\begin{array}{l}0.01 \\
\overline{0.15} \\
= \\
\overline{0} \\
0.15 \\
0.10\end{array}$ \\
\hline Total & $\overline{5.96}$ & $\overline{2.81}$ & $\overline{9.57}$ & $\overline{5.30}$ & $\overline{3.92}$ & $\overline{2.45}$ & $\overline{9.84}$ & $\overline{17.31}$ & $\overline{13.80}$ & $\overline{3.30}$ & $\overline{4.26}$ & $\overline{8.43}$ & $\overline{9.55}$ & $\overline{3.41}$ \\
\hline
\end{tabular}


Table 4. Average concentrations of THAA, DFAA, and DCAA in interstitial waters from Hole 695A. (Unit: $\mu \mathrm{mol} / \mathbf{L}$ )

\begin{tabular}{|c|c|c|c|}
\hline & THAA & DFAA & DCAA \\
\hline Acidic & 1.34 & 0.21 & 1.13 \\
\hline Aspartic acid & 0.37 & 0.17 & 0.20 \\
\hline Glutamic acid & 0.97 & 0.04 & 0.93 \\
\hline Basic & 1.09 & 0.79 & 0.30 \\
\hline Ornithine & 0.69 & 0.51 & 0.18 \\
\hline Lysine & 0.23 & 0.13 & 0.10 \\
\hline Histidine & 0.00 & 0.00 & - \\
\hline Arginine & 0.16 & 0.14 & 0.02 \\
\hline \multicolumn{4}{|l|}{ Neutral } \\
\hline hydroxy & 3.93 & 2.56 & 1.38 \\
\hline $\begin{array}{l}\text { Threonine } \\
\text { Serine }\end{array}$ & 0.26 & 0.18 & 0.08 \\
\hline straight & 1.28 & 0.93 & 0.35 \\
\hline $\begin{array}{l}\text { Glycine } \\
\text { Alanine }\end{array}$ & 1.31 & 0.74 & 0.57 \\
\hline branch & 0.55 & 0.36 & 0.19 \\
\hline Valine & 0.11 & 0.05 & 0.06 \\
\hline $\begin{array}{l}\text { Iso-leucine } \\
\text { Leucine }\end{array}$ & 0.13 & 0.09 & 0.04 \\
\hline The rest & 0.17 & 0.09 & 0.08 \\
\hline Aspargine & 0.13 & 0.12 & 0.01 \\
\hline Aromatic & 0.50 & 0.41 & 0.09 \\
\hline Tyrosine & 0.30 & 0.29 & 0.01 \\
\hline Phenylalanine & 0.20 & 0.12 & 0.08 \\
\hline Sulfur & 0.02 & 0.01 & 0.01 \\
\hline \multicolumn{4}{|l|}{ Methionine } \\
\hline The rest & 0.02 & 0.01 & 0.01 \\
\hline Taurine & 0.03 & 0.02 & 0.01 \\
\hline Citrulline & - & - & - \\
\hline Crytathionine & 0.04 & 0.01 & 0.03 \\
\hline $\begin{array}{l}\alpha \text {-aminoadipic acid } \\
\alpha \text {-aminobutyric }\end{array}$ & 0.03 & 0.03 & - \\
\hline acid & - & - & - \\
\hline$\beta$-aminobutyric acid & 0.09 & 0.03 & 0.06 \\
\hline$\beta$-alanine & 0.07 & 0.00 & 0.07 \\
\hline Total & 7.14 & 4.07 & 3.07 \\
\hline
\end{tabular}

ocean water has larger percentages of alanine and the acidic amino acids than the combined amino acids. Siegel and Degens (1966) also found greater concentrations of combined than free amino acids dissolved in surface waters of Buzzard's Bay.

The reason for the dominance of DFAA over DCAA in Hole 695A is uncertain. It is possible that the DCAA we observed are actually adsorbed on or are constituents of small particles in sediments. Lee and Bada (1975) reported that glass fiber filtered $(1-2 \mu \mathrm{m})$ seawater samples have approximately three times the combined amino acid concentration of the samples filtered with Millipore $(0.45 \mu \mathrm{m})$ filters, although the concentrations of the free amino acids are identical. This would imply that the DCAA may be influenced by the amount of very small particles $(<1$ $\mu \mathrm{m})$. Our interstitial water samples were taken with $0.2 \mu \mathrm{m}$ filters, so only smaller particles could have passed through the filter. Although the interstitial water was taken by the same proce- dure in ODP Hole 677A, the ratio of combined to free amino acids in Hole 677A is different from that in Hole 695A.

The observed concentrations and composition of both DFAA and DCAA are the net result of processes that release and remove them from interstitial waters and hence are kinetically controlled. Thus the production and decomposition rates of amino acids should be studied.

\section{Combined Amino Acids in Interstitial Waters in Lithologic Units I and II}

Four possible constituents make up the DCAA: (1) small flakes of marine biological material; (2) small particles of terrigenous material; (3) the decomposition products of cellular material and humic acid-like materials dissolved in interstitial water; and (4) small inorganic particles on which combined organic materials are adsorbed.

Based upon smear slide examination, biogenic siliceous materials including diatoms, radiolarians, and sponge spicules constitute about $50 \%$ and $20 \%$ of sediments in Units I and II, respectively. Diatoms make up $90 \%$ of some samples in Unit I. Therefore siliceous plankton is considered to be the major initial contributor to the protein amino acids of sediments. Diatoms are enriched in aspartic acid, serine, proline, glutamic acid, glycine, and alanine whose relative abundance is each more than $10 \%$ of total amino acids (Degens, 1976). The amino acid composition of sponges is almost identical to that of diatoms (Degens, 1970). Marine particulate matter is enriched in glycine, glutamic, and aspartic acids. Aspartic acid content is usually equal to glutamic acid content in marine plankton and marine suspended matter (Siezen and Mague, 1978). River suspensions which originated from terrigenous materials show predominance of aspartic and glutamic acids, glycine and alanine (Peake et al., 1972).

The major DCAA found in this study are glutamic acid, serine, glycine, alanine, and aspartic acid. Glutamic acid constitutes as much as $30 \%$ of the total combined amino acids. Except for glutamic acid, the major constituent amino acids in DCAA are comparable to those present in diatom and other siliceous plankton. Thus much of the DCAA in interstitial waters originally is derived from siliceous plankton. However, other sources enriched in glutamic acid contribute to the DCAA composition.

\section{DFAA in Interstitial Waters}

The acidic amino acid fraction shows a strong correlation with the neutral amino acid fraction (Fig. 3A). The correlation coefficient is 0.99 . The ratio of acidic to neutral amino acids is 0.088. Data on DFAA from Lithologic Units I, II, and III fall on the same line in spite of the variable concentrations. The basic amino acids correlate with the neutral amino acids (Fig. 3B). The correlation coefficient is 0.91 . The ratio of basic to neutral amino acids is 0.24 .

The results of DFAA analyses are different from those of DCAA (Fig. 4A). The data show a positive, weak correlation between neutral and acidic amino acids. The correlation coefficient is 0.37 . The basic amino acids have no correlation with neutral amino acids (Fig. 4B).

Glutamic acid makes up only $0.8 \%$ of the total DFAA while its relative abundance is $12.8 \%$ of the total THAA in interstitial waters. The much lower abundance of glutamic acid in DFAA is in contrast to its high relative abundance in DFAA of interstitial waters from anoxic sediments. The possible mechanisms for large sinks of acidic amino acid, especially glutamic acid, are (1) the reaction between amino acids and clay minerals or carbonate, (2) microbial uptake and removal, and (3) the reaction between amino acids and organic materials: 
A

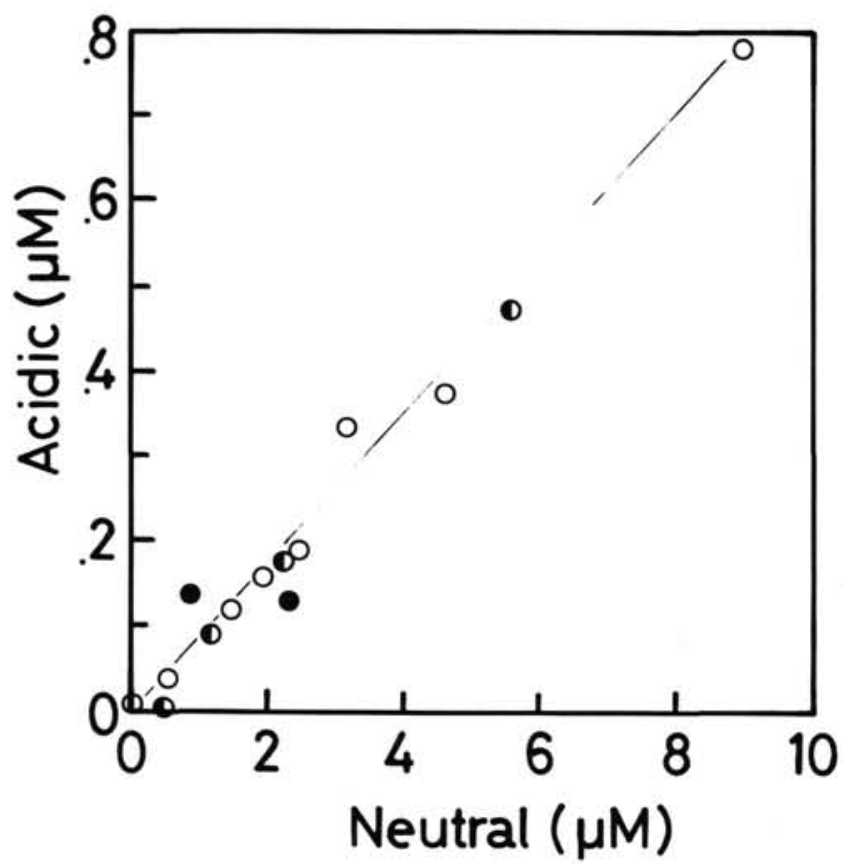

B

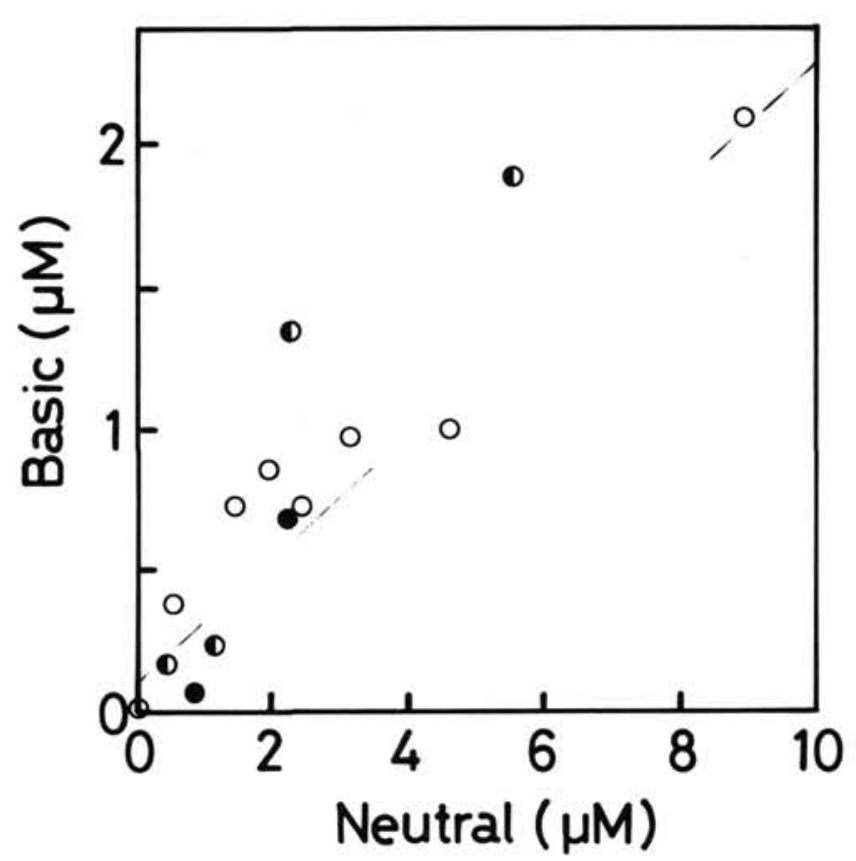

Figure 3. A. Acidic amino acids vs. neutral amino acids of DFAA from Hole 695A. B. Basic amino acids vs. neutral amino acids of DFAA from Hole 695A. (open circle $=$ Lithologic Unit I; half solid circle = Unit II; solid circle = Unit III).

A

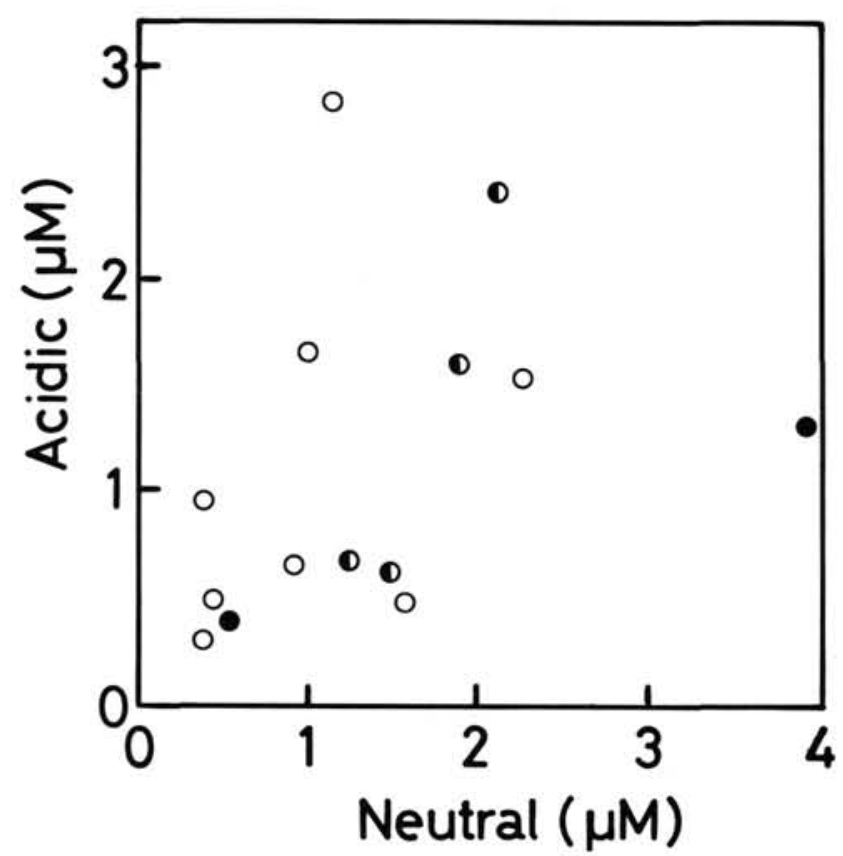

B

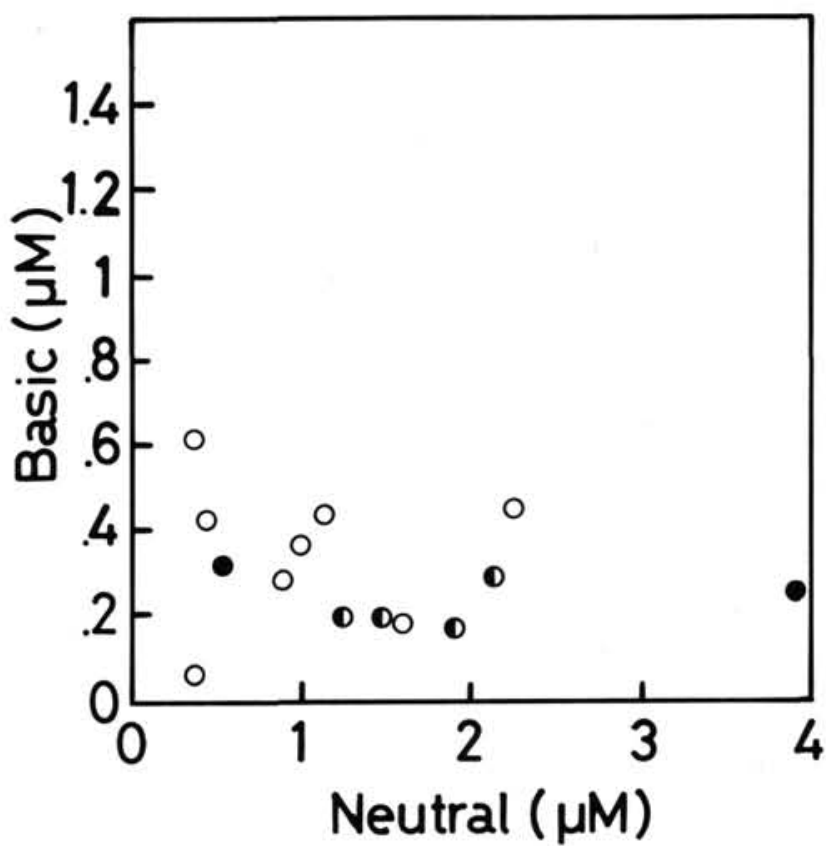

Figure 4. A. Acidic amino acids vs. neutral amino acids of combined amino acids fraction from Hole 695A. B. Basic amino acids vs. neutral amino acids of combined amino acid fraction from Hole 695A. (Open circle = Lithologic Unit I; Half solid circle = Unit II; Solid circle = Unit III). 
1. Since the sediments are composed of abundant inorganic materials (e.g., clays and minerals), the reaction between amino acids and inorganic materials is the most possible mechanism. About $40 \%$ of added, radiolabeled alanine is rapidly (within minutes) adsorbed by coastal marine sediments and subsequently decomposed much more slowly than dissolved alanine (Cristensen and Blackburn, 1980). Adsorption to montmorillonite and kaolinite inhibits utilization of amino acids and peptides by several soil microorganisms (Dashman, 1977). It is suggested that there is a limited number of available binding sites on sediment particles because of the decrease of relative adsorption with increasing amino acid and ammonium concentrations (Henrichs and Farrington, 1987). A basic amino acid (lysine) reacted much more rapidly than neutral (valine) or acidic (glutamic acid) amino acid with glucose to form melanoidin-type polymers and lysinecontaining polymers were adsorbed by clay minerals to a much greater extent than other types (Hedges, 1978). The basic amino acid fraction has a net positive charge at around $\mathrm{pH} 7$, while the acidic fraction is negatively charged and the neutral fraction is neutral. Positively charged species would interact with the negatively charged exchange sites provided by clay minerals and humic substances in sediments (Parks, 1975; Schnitzer and Khan, 1972), whereas adsorption sites for neutral and negative amino acids would be more limited (Hedges, 1978). Abelson and Hare (1971) found slight $(10 \%-30 \%)$ uptake of acidic and neutral amino acids by kerogens and humic substances from $100 \mu \mathrm{mol} / \mathrm{L}$ solutions, but substantial uptake $(70 \%-100 \%)$ of basic amino acids. The concentration of the basic fraction of DFAA is identical to that of DCAA. Therefore clay minerals did not seriously interfere with the amino acid composition although the sediments from Hole 677A contain appreciable amount of clay minerals.

Kawahata and Ishizuka (1989) interpreted the lower relative abundance of acidic fractions of DFAA than THAA in interstitial water from Hole $677 \mathrm{~A}$ as due to the adsorption or reaction with carbonates which are abundant in the sediments. Since the abundance of biogenic or inorganic carbonates is quite low (less than $2 \%$ ) in Hole 695A, carbonates could not control the acidic fraction in interstitial waters. Quartz, which is the second main constituent in sediments from Hole $695 \mathrm{~A}$ based upon microscopic observation, does not react with interstitial waters.

2. Sulfate reduction is found below $50 \mathrm{~m}$ sub-bottom depth. Biological methane occurs below $250 \mathrm{~m}$ sub-bottom depth. In addition, high concentrations of ammonia and phosphate in interstitial waters imply high microbial activity in Hole 695A. The clear relation between amino acid compositions and the type of microbial activity (e.g., sulfate reduction and methane fermentation) can not be identified when the concentration and composition profiles of DFAA and DCAA are compared with the profile of sulfate, methane, ammonium, and phosphate. Garder and Hanson (1979), Jorgensen et al. (1981), Burgdige and Martens (1987), Jorgensen (1987), and Henrichs and Farrington $(1984,1987)$ reported that glutamic acid is often a major constituent although concentrations of other amino acids, including alanine, glycine, aspartic acid, asparagine, serine, and ornithine are sometimes greater than or equal to glutamic acid concentration. Garder and Hanson (1979) suggest that bacteria may produce high relative levels of glutamic acid and alanine in DFAA in interstitial waters of Georgia Salt Marsh soils. Henrichs and Farrington (1984) also suggest a bacterial source for most of the DFAA in sediments where sulfate reduction occurs. DFAA in interstitial water from anoxic sediments are commonly enriched in glutamic acid while our samples are depleted. Since there is little known about amino acid excretion by bacteria in marine environments, the possibility cannot be eliminated of important microbial uptake and removal of glutamic acid.
3. Rosenfeld (1979) found that glutamic acid and ornithine were adsorbed more strongly by clay sediments than $\alpha$-aminobutyric acid. Adsorption decreased after peroxide treatment, which may indicate that organic matter was a major adsorber. The sediments recovered from Hole 695A show high organic content. Although we didn't analyze humic substances in the sediments, organic material may affect the DFAA composition in the interstitial waters.

\section{ACKNOWLEDGMENTS}

The authors wish to acknowledge Drs. I. Koike and M. Ohmori for providing technical advice on the analytical procedure. Also they are grateful to on-board technicians of the Ocean Drilling Program for squeezing interstitial waters from sediments. This work was supported by a Research Grant of the Agency of Industrial Science and Technology and the Ministry of Education, Japan.

\section{REFERENCES}

Abelson, P. H., and Hare, P. E., 1971. Reaction of amino acids with natural and artificial humus and kerogens. Carnegie Inst. Wash. Yearb., 69:327-334.

Burgdige, D., and Martens, C. S., 1984. Amino acid cycling in organicrich marine sediments. EOS, Trans. Am. Geophys. Union., 65:960.

Cristensen, D., and Blackburn, T. H., 1980. Turnover of tracer $\left({ }^{14} \mathrm{C},{ }^{3} \mathrm{H}\right.$ labelled) alanine in shore marine sediments. Mar. Biol., 58:97-103.

Dashman, T., 1977. Adsorption of amino acids and peptides on homoionic montmorillonite and kaolinite, and the utilization of the adsorbed amino acids and peptides by microorganisms. [Ph.D.dissert.], New York Univ.

Degens, E. T., 1970. Molecular nature of nitrogenous compounds in seawater and recent marine sediments. In Hood, D. W. (Ed.), Symposium on Organic Matter in natural waters, Inst. Mar. Sci. (Alaska), Occas. Publ. 1:77-106.

Garder, W. S., and Hanson, R. B., 1979. Dissolved free amino acids in interstitial water of Georgia salt marsh soils. Estuaries, 2:113-118.

Hedges, J., 1978. The formation and clay mineral reactions of melanoidins. Geochim. Cosmochim. Acta, 42:69-76.

Henrichs, S. M., and Farrington, J. W., 1987. Peru upwelling sediments near $15^{\circ} \mathrm{S}, 1$. Remineralization and accumulation of organic matter. Limnol. Oceanogr., 29:20-34.

Henrichs, S. M., and Farrington, J. W., 1987. Early diagenesis of amino acids and organic matter in two coastal marine sediments. Geochim. Cosmochim. Acta., 51:1-15.

Ishizuka, T., Nozaki, Y., and Shimooka, K., 1988. Amino acids in the interstitial waters of ESOPE long cores from two North Atlantic abyssal plains. Geochemical J., 22:1-8.

Jorgensen, N.O.G., 1984. Microbial activity in the water-sediment interface: assimilation and production of dissolved free amino acids. Oceanis, 10:365-377.

Jorgensen, N.O.G., Lindroth, P., and Mopper, K., 1981. Extraction and distribution of free amino acids and ammonium in sediments and overlying sea waters from the Limfjord, Denmark. Oceanol. Acta, 4:465-474.

Kawahata, H., and Ishizuka, T., 1989. Organic property of sediments and amino acids in interstitial waters from the flank of Costa Rica Rift, Galapagos Spreading Center (ODP Sites 677 and 678). In Becker, K., Sakai, H., et al., Proc. ODP, Sci. Results, 111: College Station, TX (Ocean Drilling Program).

Lee, C., and Bada, J. L., 1975. Amino acids in equatorial Pacific Ocean water. Earth Planet. Sci. Lett., 26:61-68.

Manheim, F. T., and Schug, D. M., 1978. Interstitial waters of Black Sea cores. In Ross, D. A., Neprochnov, Y. P., et al., Init. Repts. DSDP, 42, Pt. 2. Washington (U.S. Govt. Printing Office), 637-651.

Michaelis, W., Mycke, B., Vogt, J., Schuetze, G., and Degens, T. 1982. Organic geochemistry of interstitial waters, Sites 474 and 479, Leg 64. In Curray, J. R., Moore, D. G., et al., Init. Repts. DSDP, 64, Washington (U.S. Govt. Printing, Office), 933-937.

Parks, G., 1975. Adsorption in the marine environment. In Riley J. P., Skirrow, G., (Eds.), Chemical Oceanography, 2nd ed. (Academic Press) New York, 1:271-308. 
Peake, E. P., Baker, B. L., and Hodgson, G. W., 1972. Hydrogeochemistry of the surface waters of the Mackenzie River drainage basin, Canada-II. The contribution of amino acids, hydrocarbons and chlorine to the Beaufort Sea by the Mackenzie River system. Geochim. Cosmochim. Acta, 36:867-883.

Rosenfeld, J. K., 1979. Amino acid diagenesis and adsorption in nearshore anoxic sediments. Limnol. Oceanogr., 24:1014-1021.

Schnitzer, M., and Khan, S. U., 1972. Humic Substances in the Environment. New York (Marcel Dekker).

Shipboard Scientific Party, 1988. Site 695. In Barker, P. F., Kennett, J. P., et al., Proc. ODP, Init. Repts., 113: College Station, TX (Ocean Drilling program), 527-606.
Siegel, A., and Degens, E. T., 1966. Concentration of dissolved amino acids from saline waters by ligand-exchange chromatography. Science, 151:1098.

Siezen, R. J., and Mague, T. H., 1978. Amino acids in suspended particulate matter from oceanic and coastal waters of the Pacific. Mar. Chem., 6:215-231.

Date of initial receipt: 15 November 1988

Date of acceptance: 21 July 1989.

Ms 113B-166 